\title{
Editorial
}

\section{Recent Advances on the Theory and Applications of Hybrid Systems}

\author{
Rui Wang, ${ }^{1}$ Guo-Ping Liu, ${ }^{2}$ Yun-Bo Zhao, ${ }^{3}$ and Weiguo Xia ${ }^{4}$ \\ ${ }^{1}$ School of Aeronautics and Astronautics, Dalian University of Technology, Dalian 116024, China \\ ${ }^{2}$ Faculty of Advanced Technology, University of South Wales, Pontypridd CF37 1DL, UK \\ ${ }^{3}$ Department of Automation, Zhejiang University of Technology, Hangzhou 310023, China \\ ${ }^{4}$ ACCESS Linnaeus Centre, School of Electrical Engineering, Royal Institute of Technology (KTH), 10044 Stockholm, Sweden
}

Correspondence should be addressed to Rui Wang; ruiwang@dlut.edu.cn

Received 24 May 2016; Accepted 24 May 2016

Copyright (C) 2016 Rui Wang et al. This is an open access article distributed under the Creative Commons Attribution License, which permits unrestricted use, distribution, and reproduction in any medium, provided the original work is properly cited.

Hybrid systems are dynamical systems with interactions of both continuous and discrete dynamics [1]. Such systems can be appropriate models for a multitude of practical systems in engineering and applied sciences $[2,3]$. From the control perspective, a typical example of hybrid systems would consist of a continuous-time linear time-invariant plant described by linear differential equations and a discretetime linear time-invariant controller described by linear difference equations, where the former involves continuousvalued variables that depend on the continuous time, and the latter involves continuous-valued variables that depend on the discrete time, thus the hybrid feature of the whole system [4].

Since the interactions of continuous-time and discretetime dynamics in a hybrid system lead to rich dynamical behaviours which may never be encountered in purely continuous-time systems, hybrid systems thus stand as a highly challenging field of research, encompassing a variety of challenging problems in many aspects such as modeling, simulation, analysis, verification, and controller synthesis $[3,5]$. In view of the facts that hybrid systems modeling is popular in practice, their design and analysis are therefore of great importance not only in theory but also in practice. We hence felt urged to provide a discussion platform for the researchers to report their state-of-the-art works in this area, which eventually motivated the organization of this special issue on the recent advances of the theory and applications of hybrid systems.
In this special issue we have gathered a number of research works on hybrid systems and the closely related topics. We are very happy to see that these limited numbers of research works have shown a great variety, in a sense giving a fairly complete picture of the state-of-the-art research on hybrid systems.

The works included in this special issue can be roughly divided into two categories, one focusing on the control strategy design and the other on the system performance analysis of hybrid systems.

On the one hand, various control strategies for hybrid systems have been discussed in this special issue. These control strategies include, for example, an improved average dwell method for the stabilization of a class of switched system (Q. Su and P. Wang), $H_{\infty}$ output tracking algorithm for switched cascade nonlinear systems (X. Dong et al.), a global eco-driving optimal control strategy for bus transit to minimize the exhaust emission (X. Zheng and L. Zhang), a consensus algorithm under directed switching communication topologies for linear multiagent systems (Y. Zhang et al.), and a control strategy of shifting time involving multigroup clutches for a hydromechanical continuously variable transmission (Z. Zhu et al.).

On the other hand, system performance evaluations are also widely discussed in this special issue. These works include, for example, a new performance index for hybrid energy storage system in a parallel hybrid electrical vehicle (C. Zhang et al.), the convergence analysis for a class of 
periodic boundary value problem of hybrid system with causal operators (P. Wang et al.), the limit cycle properties for both uncoupled and coupled hybrid systems (A. FavelaContreras et al.), a numerical safety evaluation method based on a compositional barrier certificate (G. Wang et al.), and the $L_{2}$-gain analysis of hybrid systems (T. Osuna et al.), just to name a few.

To conclude, we believe that this special issue contains sufficiently interesting materials on hybrid systems as well as certain related topics, both in theory and in applications. We hope that people will find this special issue a useful reference for their future works on hybrid systems.

\author{
Rui Wang \\ Guo-Ping Liu \\ Yun-Bo Zhao \\ Weiguo Xia
}

\title{
References
}

[1] J. Lygeros, K. H. Johansson, S. N. Simić, J. Zhang, and S. S. Sastry, "Dynamical properties of hybrid automata," IEEE Transaction on Automatic Control, vol. 48, no. 1, pp. 2-17, 2003.

[2] M. Bernardo, U. Montanaro, and S. Santini, "Hybrid model reference adaptive control of piecewise affine systems," IEEE Transactions on Automatic Control, vol. 58, no. 2, pp. 304-316, 2013.

[3] P. J. Antsaklis and A. Nerode, "Hybrid control systems: an introductory discussion to the special issue," IEEE Transaction on Automatic Control, vol. 43, no. 4, pp. 457-460, 1998.

[4] P. J. Antsaklis, "A brief introduction to the theory and applications of hybrid systems," in Proceedings of the IEEE Special Issue on Hybrid Systems: Theory and Applications, vol. 88, no. 7, pp. 879-887, 2000.

[5] R. Goebel, R. G. Sanfelice, and A. R. Teel, "Hybrid dynamical systems," IEEE Control Systems, vol. 29, no. 2, pp. 28-93, 2009. 


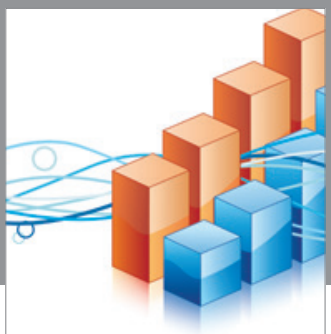

Advances in

Operations Research

vatem alat4

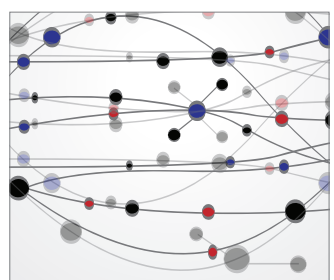

\section{The Scientific} World Journal
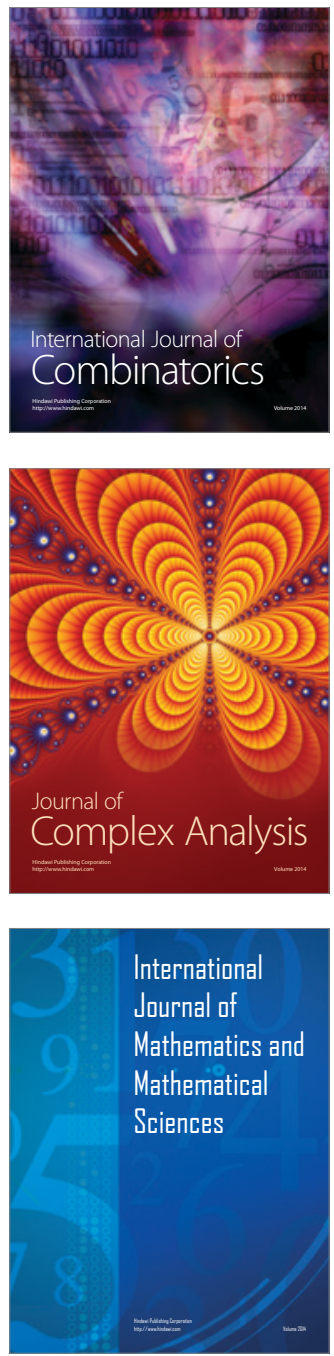
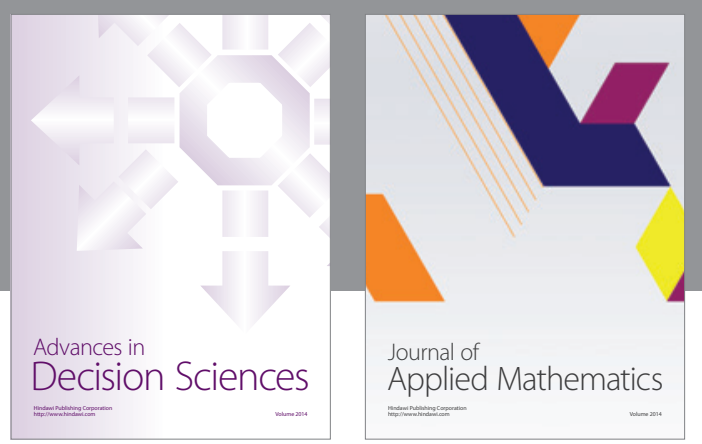

Algebra

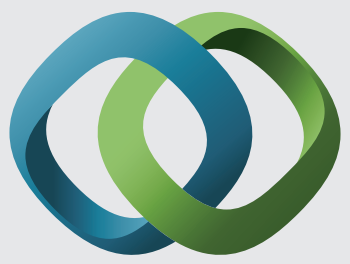

\section{Hindawi}

Submit your manuscripts at

http://www.hindawi.com
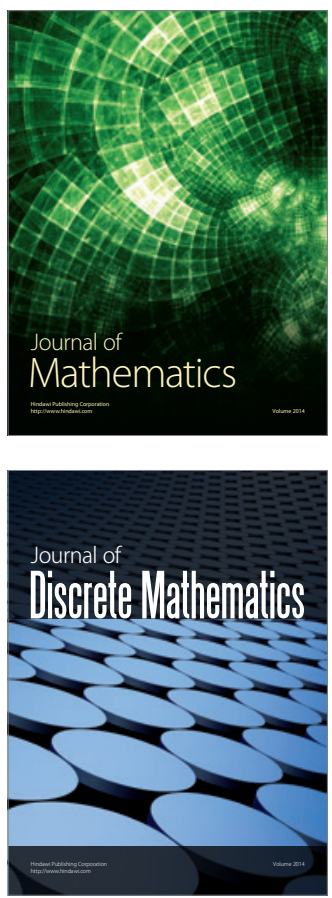

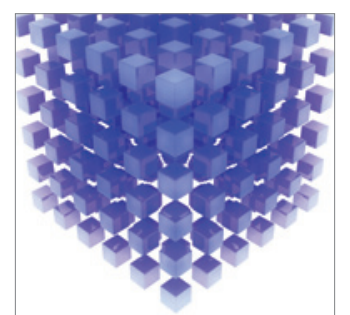

Mathematical Problems in Engineering
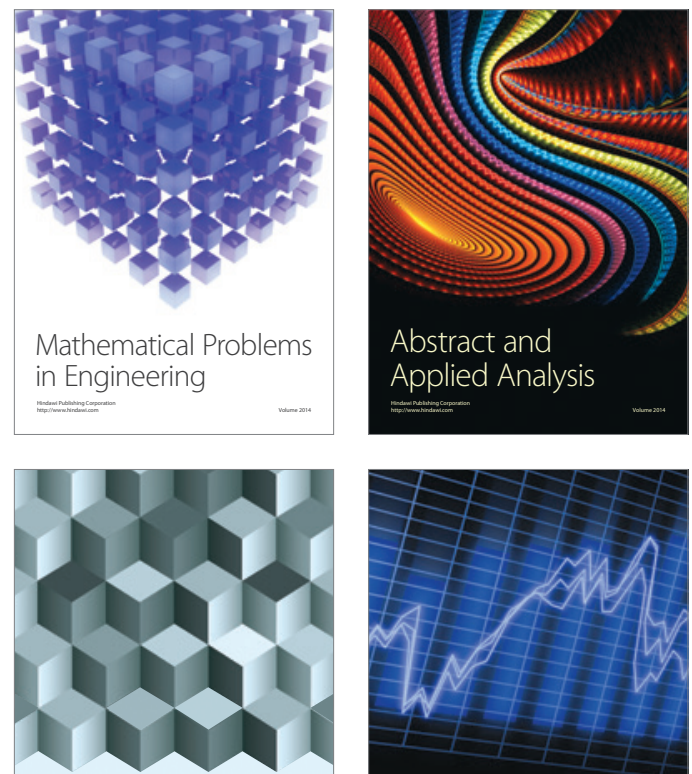

Journal of

Function Spaces

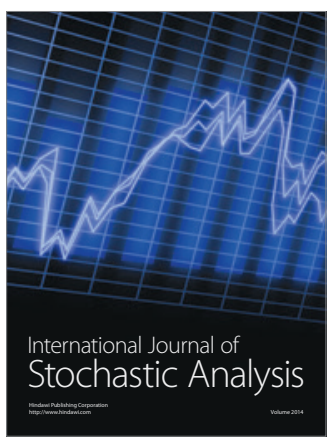

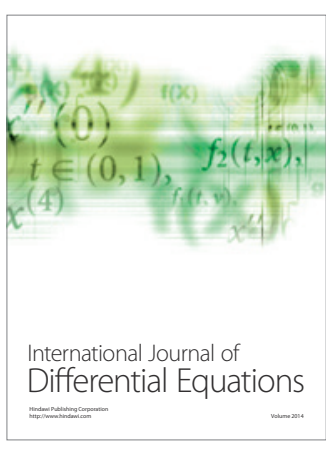
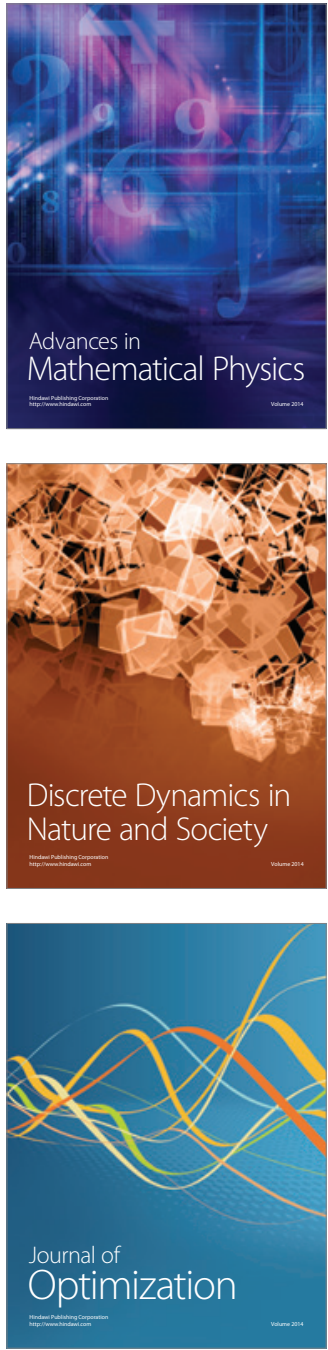